$10 \mid 2018$

Devenir écrivain

\title{
Témoignage en fragments - Kateb Yacine
}

\section{Paule Giraud}

\section{OpenEdition}

Journals

Édition électronique

URL : https://journals.openedition.org/coma/959

DOI : $10.4000 /$ coma.959

ISSN : 2275-1742

\section{Éditeur}

Institut des textes \& manuscrits modernes (ITEM)

\section{Référence électronique}

Paule Giraud, "Témoignage en fragments - Kateb Yacine », Continents manuscrits [En ligne], 10 | 2018, mis en ligne le 15 mars 2018, consulté le 13 janvier 2023. URL : http://journals.openedition.org/coma/ 959 ; DOI : https://doi.org/10.4000/coma.959

Ce document a été généré automatiquement le 13 janvier 2023.

\section{(c) (i) (9)}

Creative Commons - Attribution - Pas d'Utilisation Commerciale - Pas de Modification 4.0 International - CC BY-NC-ND 4.0

https://creativecommons.org/licenses/by-nc-nd/4.0/ 


\title{
Témoignage en fragments - Kateb Yacine
}

\author{
Paule Giraud
}

\section{NOTE DE L'ÉDITEUR}

Lorsqu'elle découvre Nedjma de Kateb Yacine, Paule Giraud, née et grandie en Auvergne, est à la veille de partir en Algérie. Cette lecture la bouleverse. «Ce que j’ai vu et vécu en Algérie après, mes contacts avec les Algériens, je l'avais déjà lu dans Nedjma [...] c'était vraiment la chair de quelqu'un qui était Algérien et qui parlait de [ce pays] comme on n'avait jamais parlé avant. " Quelques années plus tard, à son retour, elle écrit à "son écrivain ».

S'en suivra une longue amitié tissée de correspondances et de séjours que l'écrivain fera seul ou en compagnie de ses enfants, chez Paule et René Giraud dans leur maison de Blanhac en HauteLoire, se ressourçant au contact de la nature, écrivant, marchant, se réchauffant à leur amitié indéfectible.

Paule Giraud a accepté de nous offrir son témoignage sur les chemins des souvenirs ${ }^{1}$. 


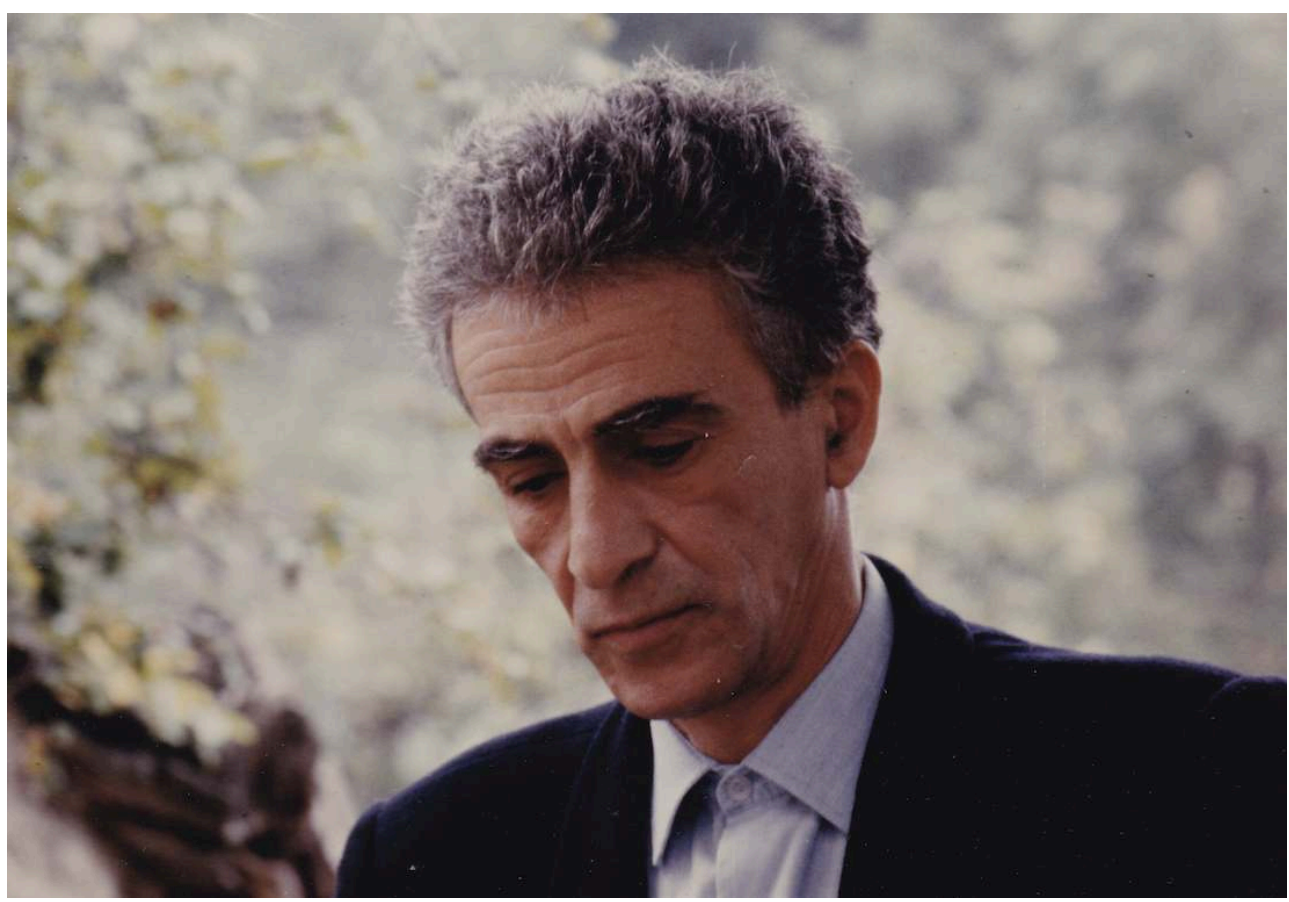

Photo René Giraud

\section{Nedjma}

1 René, mon mari, m'a offert le livre Nedjma, peu de temps après mon mariage, en me disant : il faut absolument que tu lises ce livre. Je l'ai lu, je suis vraiment entrée dedans, j'ai copié des pages entières, j'ai vraiment eu un choc, ou plusieurs chocs. Je peux résumer ma vie en disant : il y a ma vie avant le livre Nedjma et il y a ma vie après. Parce que ce livre $a$ eu une influence, Kateb a eu une influence sur moi, sur ma vie, très forte à partir de ce livre, puisque, tout ce que j'ai lu après, je ne peux pas le comparer. Voilà l'importance de Nedjma pour moi.

C'était tellement beau, je lisais et puis je copiais des pages, mais entières! Je n'en revenais pas. D'abord, la beauté de la langue, ensuite l'Algérie. Ce que j'ai vu après - je l'ai dit à Yacine [lorsque nous nous sommes rencontrés], c'est un peu pour ça que je lui ai écrit aussi - ce que j'ai vu et vécu en Algérie après, mes contacts avec les Algériens, je l'avais déjà lu dans Nedjma, mais en profondeur. Donc, ça m'a aidé à comprendre, ce n'était pas du folklore tout d'un coup, c'était vraiment la chair de quelqu'un qui était Algérien et qui parlait de ce pays comme on n'avait jamais parlé avant, je pense. Enfin j'avais déjà lu certains écrivains algériens qui étaient reconnus, avant lui ; ils écrivaient un peu comme auraient écrit des Français ou des Occidentaux sur l'Algérie. Avec Yacine, c'est l'Algérien qui a parlé. Les écrivains algériens ont senti ça, ils ont dit: on peut le faire parce qu'il l'a fait.

Voilà, je l'ai découvert comme ça. Le premier Nedjma, il a fallu le recoller parce qu'il était vraiment... 
Exemplaire de Nedjma de Paule Giraud

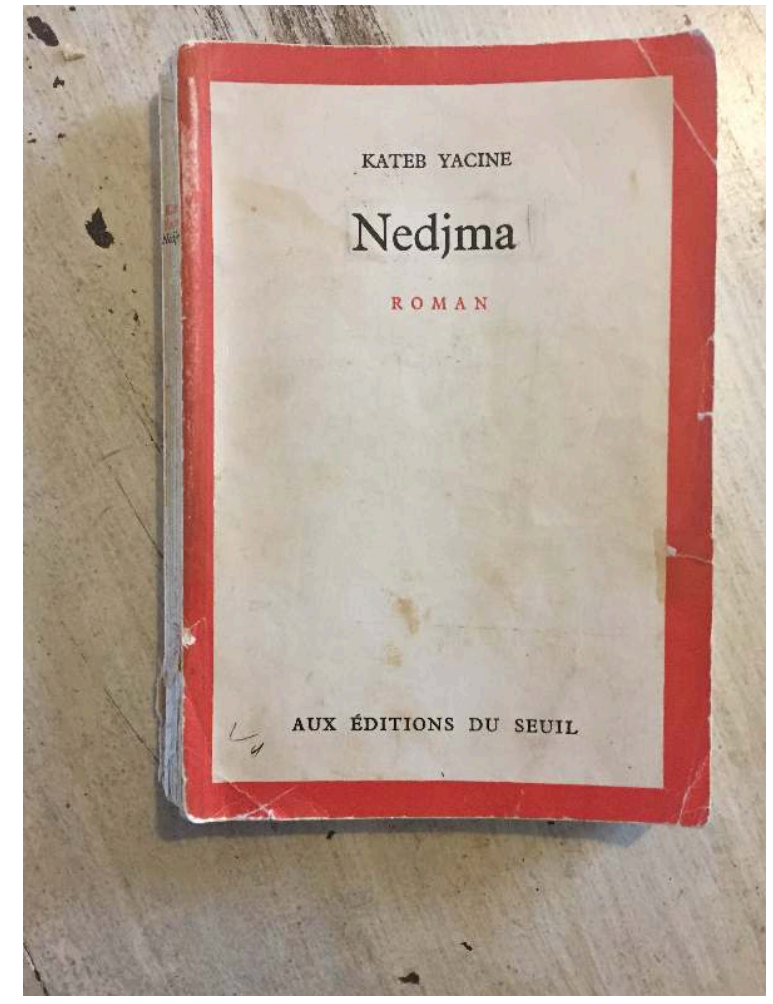

Photo Rym Khene

\section{La correspondance}

2 Notre amitié fut faite d'échanges de courriers avant la rencontre et les vacances familiales, soit à Élancourt (dans la région parisienne) soit à Blanhac. 
Quelques enveloppes des courriers adressés par Kateb Yacine aux époux Giraud

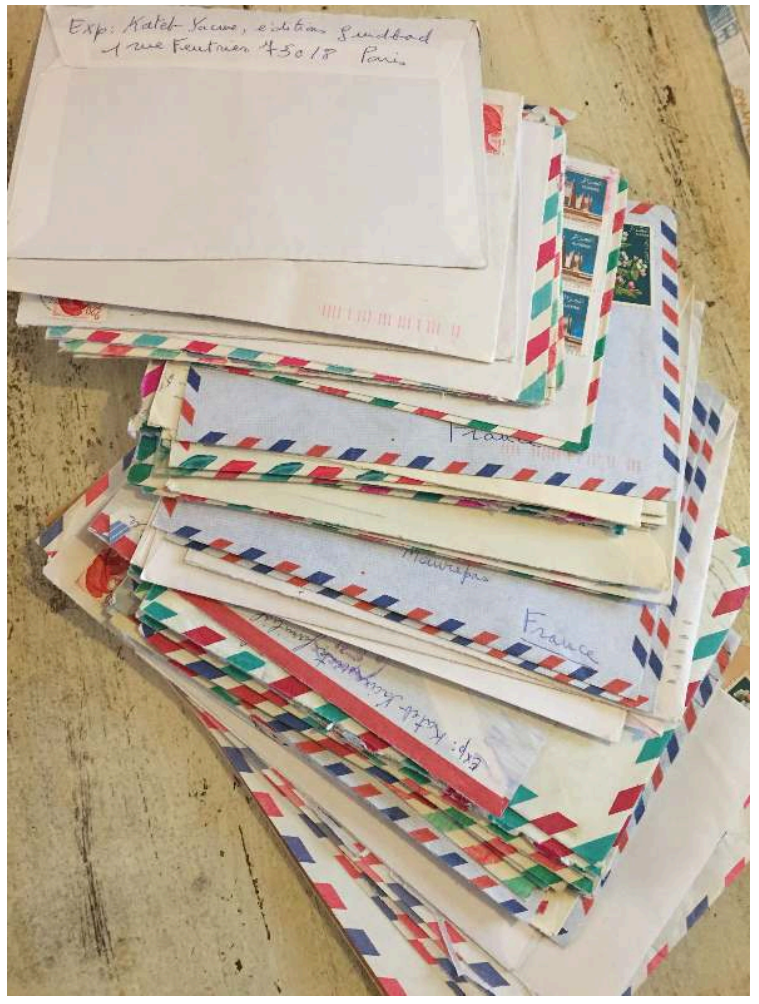

Photo Rym Khene

\section{Les habitudes d'écriture}

Kateb Yacine à la Bourgeade

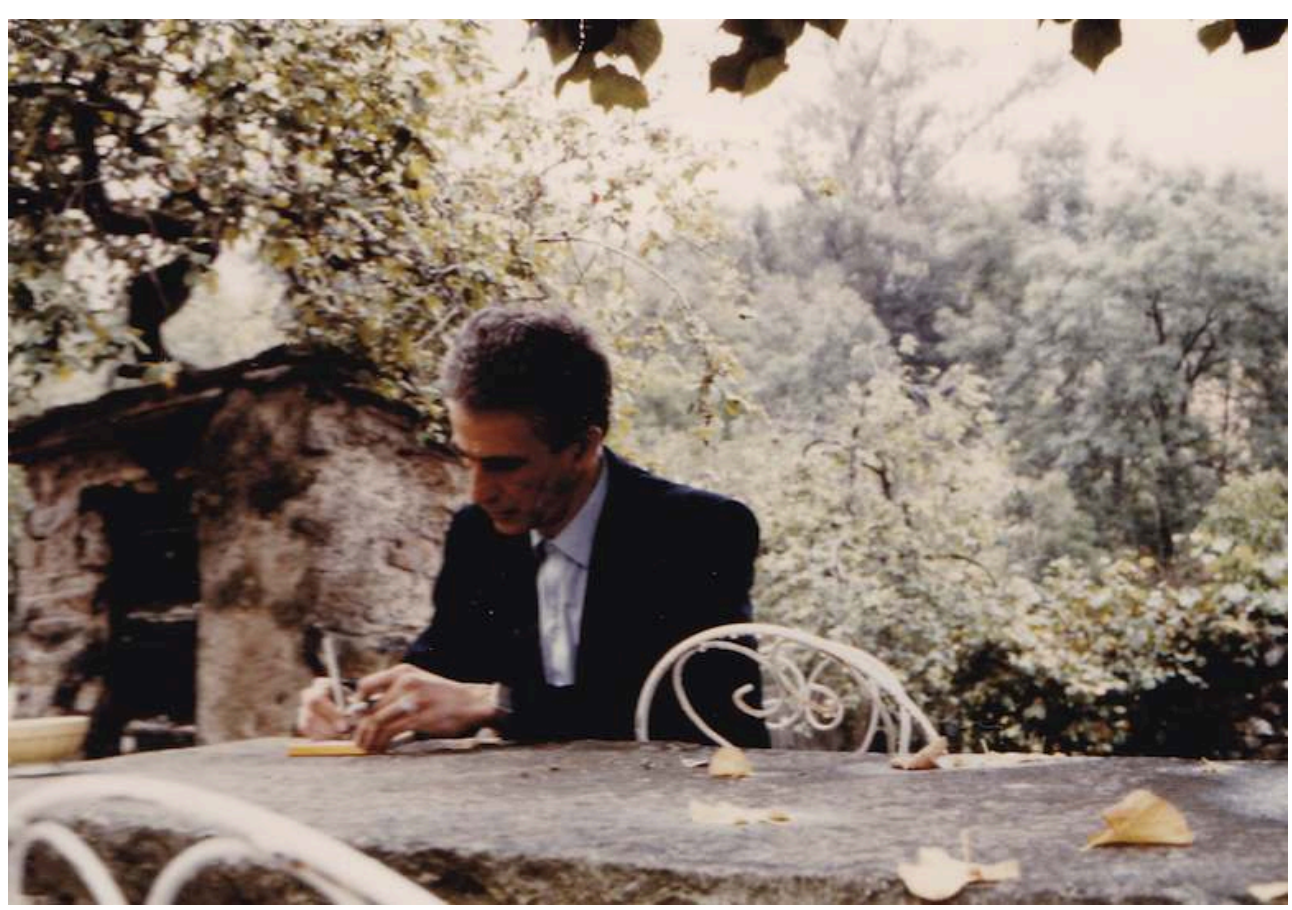

Photo René Giraud 
Quand, à quel moment de la journée écrivait-il ? C'était souvent l'après-midi, en début d'après-midi, avant de partir dans les bois. Mais le matin aussi peut-être. Il montait, il écrivait, je pense. Il regardait beaucoup dehors et il écrivait.

Où, son endroit préféré ? Sur la table en pierre, oui.

De quelle manière? Seul, entouré ? Seul, oui. Avec le chat, si possible. Il avait quelquefois le chat sur les genoux et il écrivait. Le chat voulait jouer avec le crayon, alors Yacine arrêtait d'écrire et il jouait avec le chat et avec le crayon.

Alors, ou il écrivait dans sa chambre ou dehors, mais plutôt en fin d'après-midi, sur la table en pierre.

La table de pierre de La Bourgeade

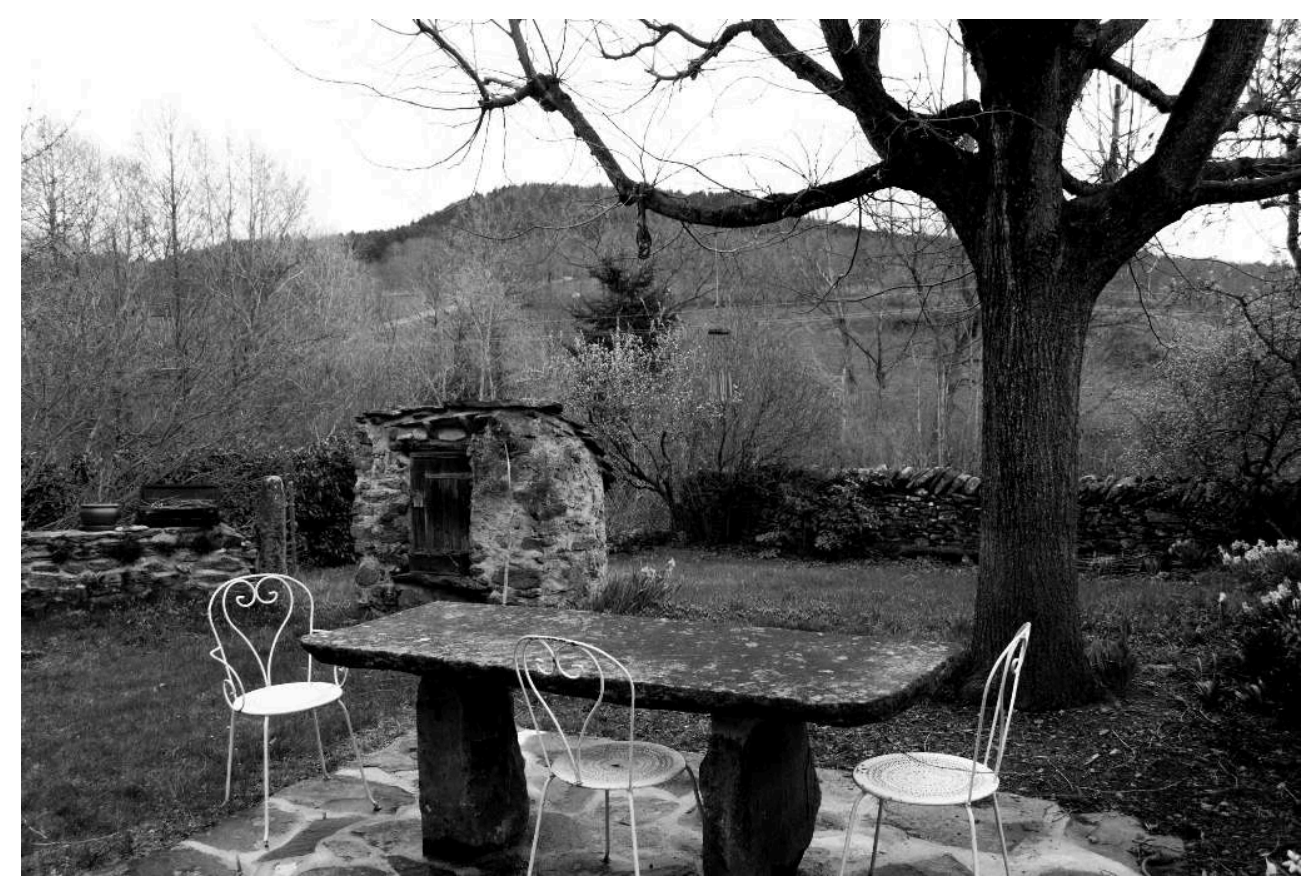

Photo Rym Khene

4 Lentement, lentement, il me semble. Il n'avait pas d'hésitations ni de précipitation, il était calme, il était bien.

Il écrivait à un moment où il était bien, peut-être avant de se promener, et, le soir, tout dépendait de son heure de retour de promenade. Il écrivait le soir dans sa chambre aussi. Plus tard, parce que, en été, il laissait les portes les fenêtres les lumières, les fenêtres ouvertes, il y avait des hannetons qui rentraient. Il ne voulait pas fermer les fenêtres. Donc, on avait essayé d'accrocher une moustiquaire, ça ne lui plaisait pas parce qu'il ne voyait plus le jardin. En fait, le jour et la nuit, quelquefois toute la nuit, c'était éclairé chez lui. Ou alors, il descendait, il faisait de la cuisine la nuit ! Donc, René descendait pour l'aider : chercher le fromage, ouvrir une boîte... Ces moments-là, de casse-croûte dans la nuit, étaient incroyablement riches de récits : séjours à l'étranger, rencontres... Yacine le faisait aussi à Élancourt. On ne voyait pas le temps passer.

\section{Arrivées à Blanhac}

La nuit, Yacine, je ne sais plus comment il dormait en fait. Il devait faire des sommes de deux heures, trois heures. En arrivant à Blanhac, parce qu'il était fatigué, il avait besoin 
de faire le point. Il disait : je remets tout à neuf. On repart, on repart à zéro. Donc, il arrivait, en train quelquefois. En général, les deux ou trois fois où il est venu par le train, il n'avait pas de valise. Il avait oublié sa valise. Une fois, il en a oublié une dans la gare à Paris. En fait, il partait sans bagages. Amazigh ${ }^{2}$ avait son sac, avec ses choses, Yacine, souvent, c'était... ses papiers quand même, il en avait dans les poches, il en avait partout. Un petit sac avec des papiers, des livres. Amazigh s'était fait très vite une bande d'amis, avec notre fille, Marie, de son âge. Ils étaient 7 ou 8, fidèles pendant presque huit ans...

\section{Des livres}

Des livres, il en laissait chez nous, mais que quelqu'un lui avait prêtés. Alors, ce qu'il emportait de la maison, on était sûrs que cela allait avoir le même sort et cela nous plaisait. Le livre était fait pour circuler, c'était ça qui était bien. Tous les jours, il fallait aller lui acheter Libé, le Monde à Rosières. Il avait lu qu'une association à Paris, dans les grandes villes, déposait des livres sur les bancs, pour que les gens les lisent. Ça, ça lui plaisait. C'est vrai, donner aux gens l'envie de lire parce qu'on voit un livre qui n'est à personne, eh bien on le prend, on le lit.

\section{Il avait tous ses papiers sur la table}

7 Lettres, journaux, écrits... Nous préparions des cahiers, des blocs, des feuilles blanches en grande quantité, pour son arrivée. Yacine écrivait partout et sur tout: sur des paquets de cigarettes parfois. Je regardais avec lui, avant de repartir, ce qu'il voulait garder ou non. Y compris dans la corbeille à papiers. Ce qu'il voulait brûler parfois. Yacine aimait le feu.

Dossiers Kateb Yacine de Paule Giraud

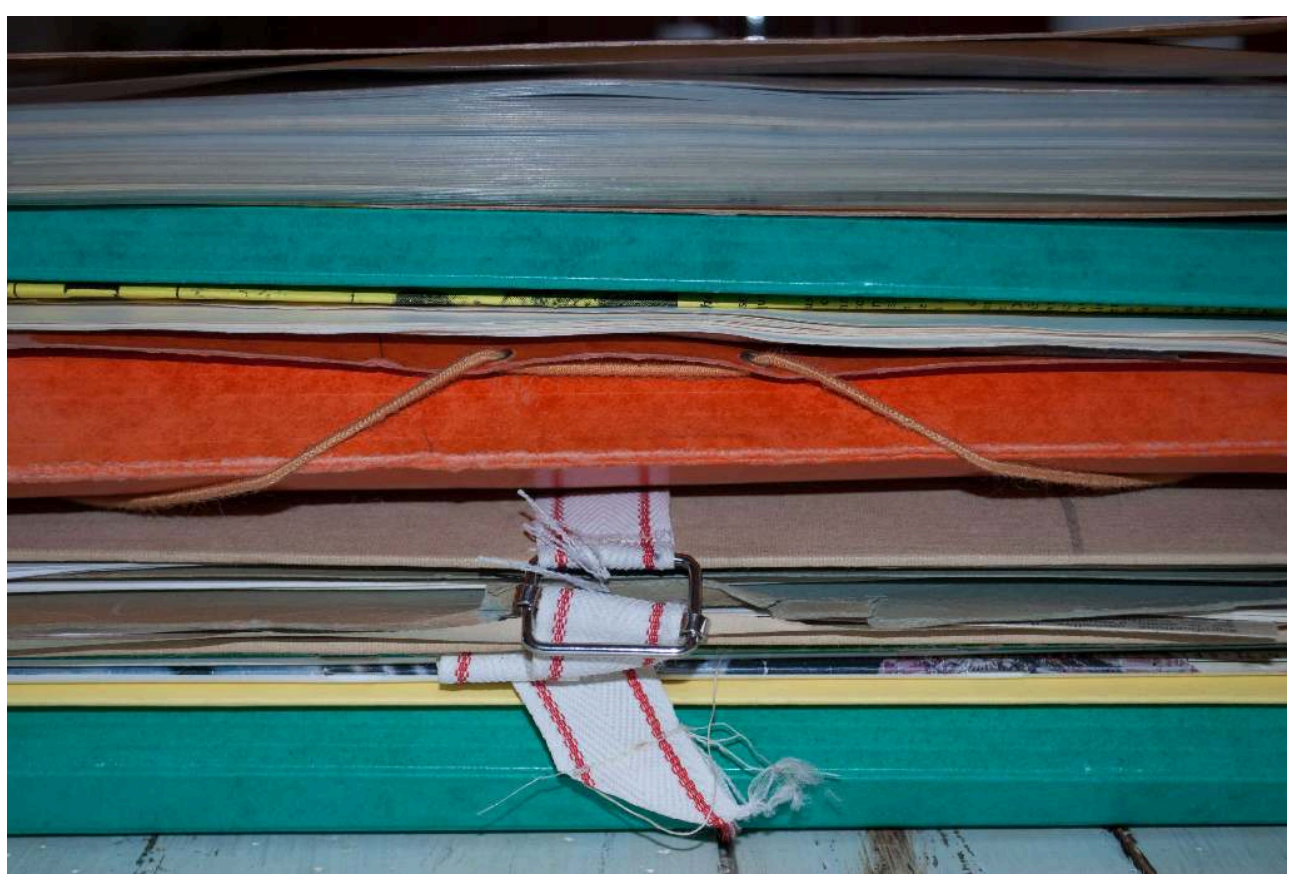

Photo Rym Khene 


\section{Il aimait le feu}

Les murs étant très épais, la grande pièce du bas était toujours très fraîche. «Un feu du tonnerre, un feu d'enfer ", Yacine aimait cela. Préparer le bois, l'âtre. Allumer le feu. Nous mettions des tables un peu partout devant, et Yacine lisait le journal, nous racontait ses séjours partout dans le monde... Nous ne voyions jamais le temps passer. Seulement les bûches à ajouter, les enfants qui venaient goûter.

Lorsqu'il y avait le feu, nous ne pouvions pas laisser Yacine seul longtemps car il aurait ajouté quatre bûches d'un coup, au moins. Cette vieille maison aurait brûlé.

Yacine nous avait raconté avoir brûlé, en plein mois d'août, la provision de bois d'amis qui lui avaient laissé leur maison normande, parce qu'il y avait une cheminée dans chaque pièce et qu'il avait froid.

Un jour où nous partions pour Paris, très tôt le matin, Yacine était triste. Il venait de perdre un ami très cher. Avant de prendre la route, il a voulu un feu dans la cheminée et nous l'avons allumé. Debout, tournant le dos au feu, Yacine me parlait de cet ami. Je le revois encore. Nous sommes partis longtemps après: "Je ne sais pas pourquoi ", avait dit Yacine, «aujourd'hui j'ai froid». Une couverture sur les épaules, il avait parcouru la moitié du chemin. Puis il s'était senti mieux soudain : «La vie est belle, le chat est du voyage !».

À la cuisine de Blanhac, il y avait un fourneau. Yacine aimait en surveiller le feu, soulever les ronds de la cuisinière, ajouter une bûche.

\section{Le chat}

9 À la maison, une fois, dans sa chambre, il y avait le chat, son chat, qui s'était couché à sa place, sur le lit, et Yacine a dormi sur le tapis. Il a tiré les coussins, il n'a pas dérangé le chat. Le lendemain, il nous a dit: Mahomet avait découpé son manteau ${ }^{3}$, je ne veux pas réveiller un chat, je peux bien dormir sur le tapis. Il a dormi sur le tapis pour ne pas réveiller le chat, mais dans la nuit, le chat s'est réveillé et est venu dormir avec lui, alors, ils sont remontés tous les deux dans le lit.

Mais, il pouvait le faire, puisque Mahomet avait fait la même chose.

\section{Les confitures}

Il aimait beaucoup la cuisine. Il aimait, pendant les vacances, qu'il y ait une femme à la cuisine. Une tante, une mère, une fille, une sœur, enfin quelqu'un qui cuisine. Il venait souvent, il soulevait les couvercles, ça lui plaisait. Yacine adorait voir les bocaux de confitures.

Et, quand il revenait de promenade, il avait quelquefois des petits bouquets, il en mettait dans un verre, c'était gentil de m'apporter un bouquet, il s'était assis, peut-être, sur le bouquet, le bouquet était comme ça, mais il était content. Alors, quand les confitures étaient là, il mettait les fleurs sur les pots. Pendant longtemps, on a gardé ces pots, on a attendu longtemps avant de les manger. Il y avait toujours une fleur, deux, trois fleurs sur le pot de confiture, avant de refermer, de mettre le couvercle ou le papier.

Voilà, il aimait qu'il y ait une cuisine dans laquelle quelqu'un s'active. Il aimait, par exemple que moi, si c'était chez moi, je cuisine ou je tricote. 


\section{Les échos avec l'Algérie}

Les échos avec l'Algérie ? C'était d'abord dans nos conversations. Et puis, quand même, dans le paysage. La Kabylie, la Haute Kabylie, il nous disait : il y a des endroits en Haute Kabylie où il y a exactement les mêmes paysages... Sûrement ! Il les connaissait mieux que nous ! Moi, je n'avais pas l'impression. Il me semblait que ce que je connaissais de la Haute Kabylie, il n'y avait pas la même végétation qu'ici.

Mais c'est surtout le village perché de Mézères qui lui plaisait. Quand il a vu Mézères, là, il s'est senti en Kabylie. "C'est un petit village berbère ", il appelait ça comme ça. «C'est mon village kabyle ou mon village berbère ».
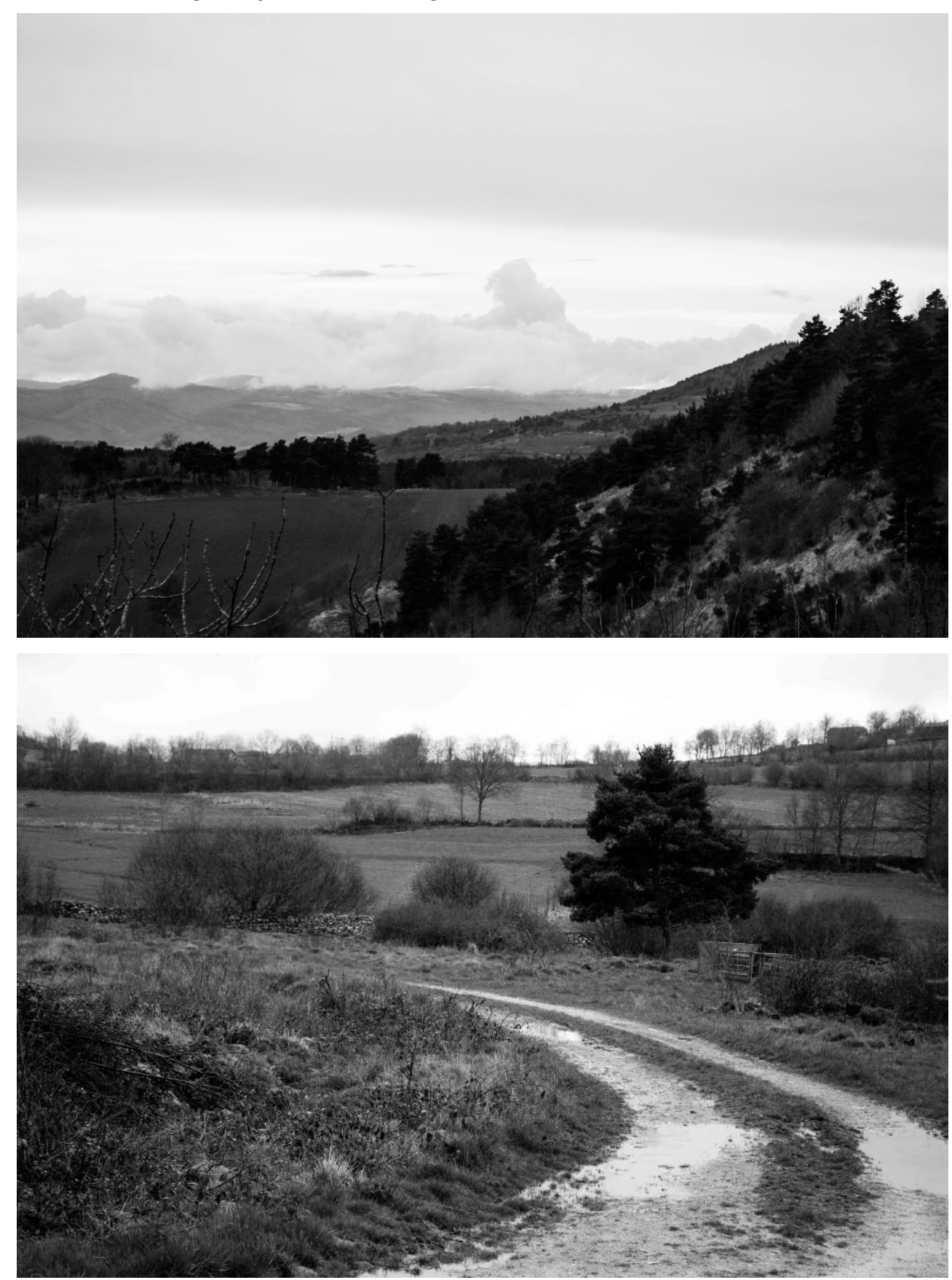

Photos Rym Khene 


\section{Promenades, son petit pré}

Il y a une petite route, qu'il aimait beaucoup, qui menait à son pré, c'est à la sortie de Blanhac. C'était un chemin à l'époque, maintenant elle est goudronnée. On sort de Blanhac, on sort des bois et on découvre Mézères et c'est ça qu'il aimait. Du pré, qu'il appelait son petit pré, on avait la même vue sur Mézères

Alors, il aimait ça, il y avait les bergers avec leurs troupeaux qui lui rappelaient l'Algérie... Il nous avait dit qu'il n'avait pas eu envie de parler d'Algérie avec les bergers, il ne voulait pas aborder ce domaine-là, ce sujet, quand il comprenait que c'étaient des gens qui avaient fait la guerre d'Algérie, il parlait d'autre chose.

Il partait en général en promenade après le repas de midi, le café, il nous disait : je vais me reposer. Il montait dans sa chambre, là, il devait écrire un peu. Mais ça ne durait pas longtemps, sauf les premiers jours où il avait besoin de dormir. Il partait se promener.

Kateb Yacine dans les bois

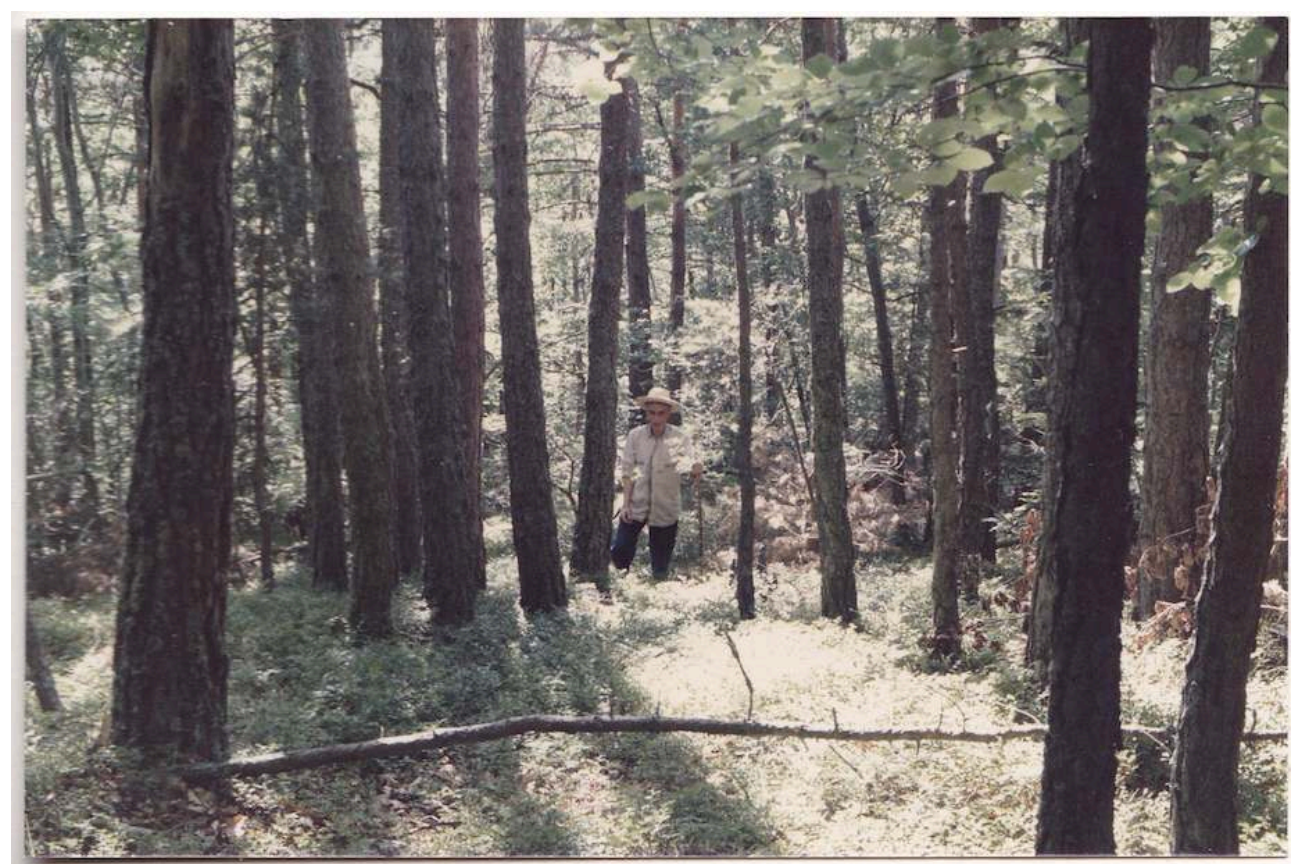

Photo René Giraud

On était inquiets parfois, parce qu'il rentrait tard. On avait peur du «Chier blanc»c'est un éboulis énorme de rochers - on y passe, mais de temps en temps, il aimait grimper sur les rochers. 
Le Chier blanc de Jalore, éboulis de roches magmatiques fragmentées

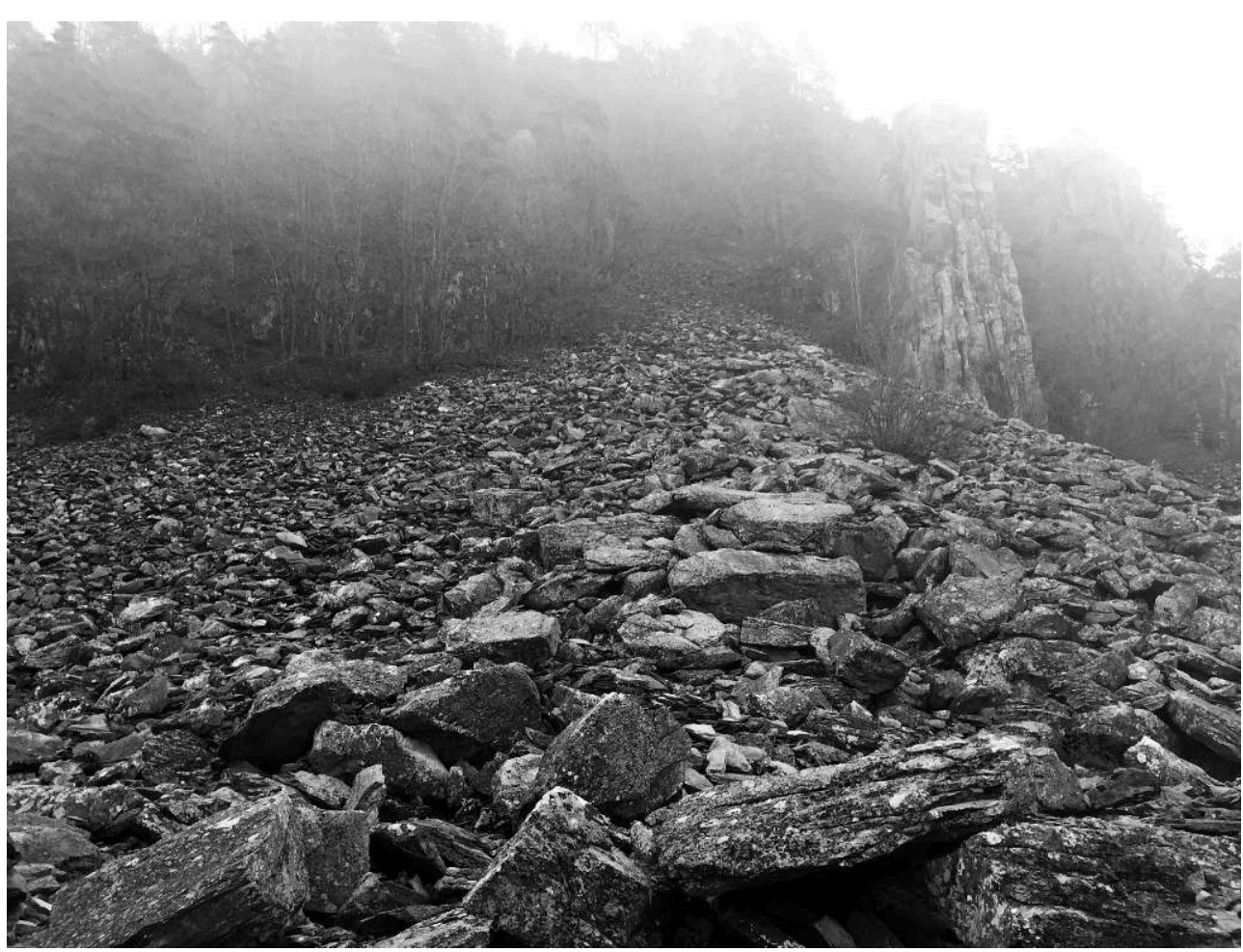

Photo Rym Khene

14 C'était un souci pour nous quand il partait se promener vers quatre heures de l'aprèsmidi, mais la plupart du temps, il partait tout l'après-midi dans les bois.

Peut-être avait-il du papier, ça, je ne sais pas, mais il n'avait pas de livre. Il partait et il nous racontait toujours quelque chose, qu'il avait vu ou entendu. Il avait vu un renard un jour, mais comme il a décrit le renard, une amie qui était là a douté : peut-être un blaireau?

Il avait remarqué que les oiseaux ne chantaient pas ; ça, ça l'avait surpris, et en fait les oiseaux chantent le matin, il l'a découvert lorsqu'il est parti se promener le matin. Le rossignol, lui, chante la nuit. De ses fenêtres là-haut, on domine la montagne, il y a un ruisseau. De sa chambre, il ne le voyait pas mais il aimait beaucoup parce qu'il y avait des rossignols. Alors la nuit, il entendait les rossignols et les chouettes. Il adorait ça. La nuit quelquefois, s'il était à sa fenêtre, c'était pour les oiseaux-

En général, quand il revenait de se promener, il avait pensé à des lectures, il avait pensé à des gens, mais à des choses paisibles. Il disait : ça m'a fait du bien, ça m'a apaisé, je me suis bien plu, je ne sais plus du tout à quoi j'ai pensé. Il en avait besoin tous les jours. 
Kateb Yacine au cours de ses promenades

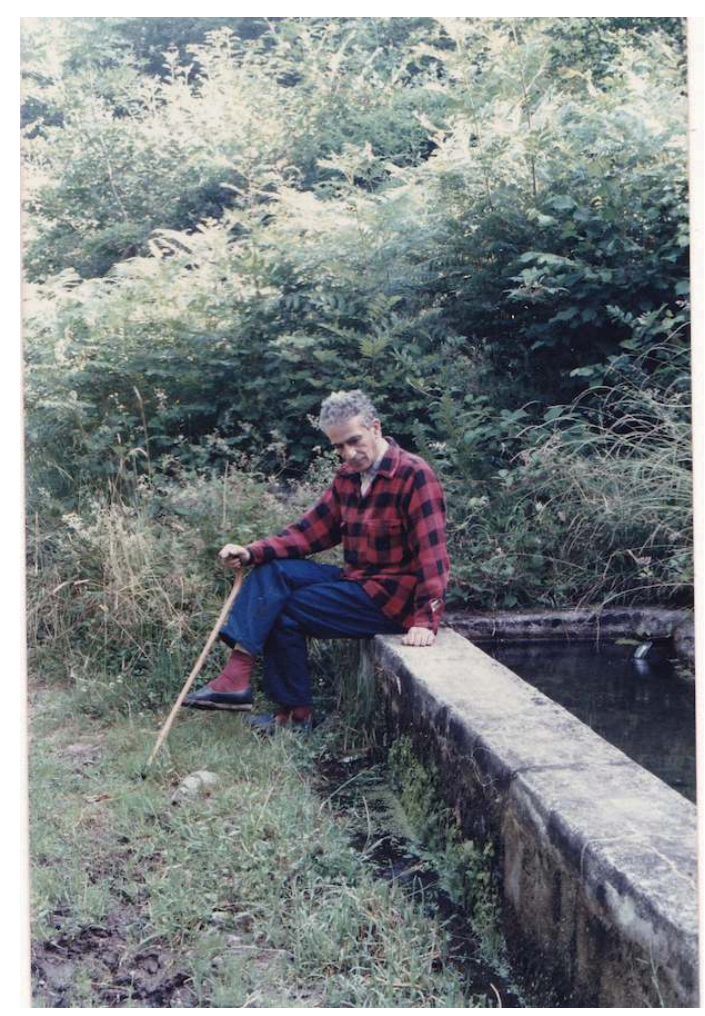

Photo René Giraud

15 Il avait découvert au coin du bois un trou, un abri de berger dans un mur. Il disait « Ma grotte ", c'était à lui. C'est tout petit en fait, il s'asseyait là, il y avait une pierre, il s'asseyait et il fumait sa cigarette. Et, quand il allait à Jalore, il y passait bien vingt trente minutes. Je pense que c'est là qu'il avait vu le renard parce qu'il ne bougeait pas. Il y avait Jalore, avec son petit bois, et la route de Mézères, avec son petit pré. C'était à lui. On disait le pré de Yacine, le bois de Yacine, le mur, l'abri... c'est resté comme ça. Ça, ça comptait, c'était une fois dans l'année, en été. Au printemps, il était chez nous, mais en région parisienne. Là, en Auvergne, c'était tout à fait différent. Il disait : je suis bien parce que je suis incognito. Personne ne viendra me chercher ici. 


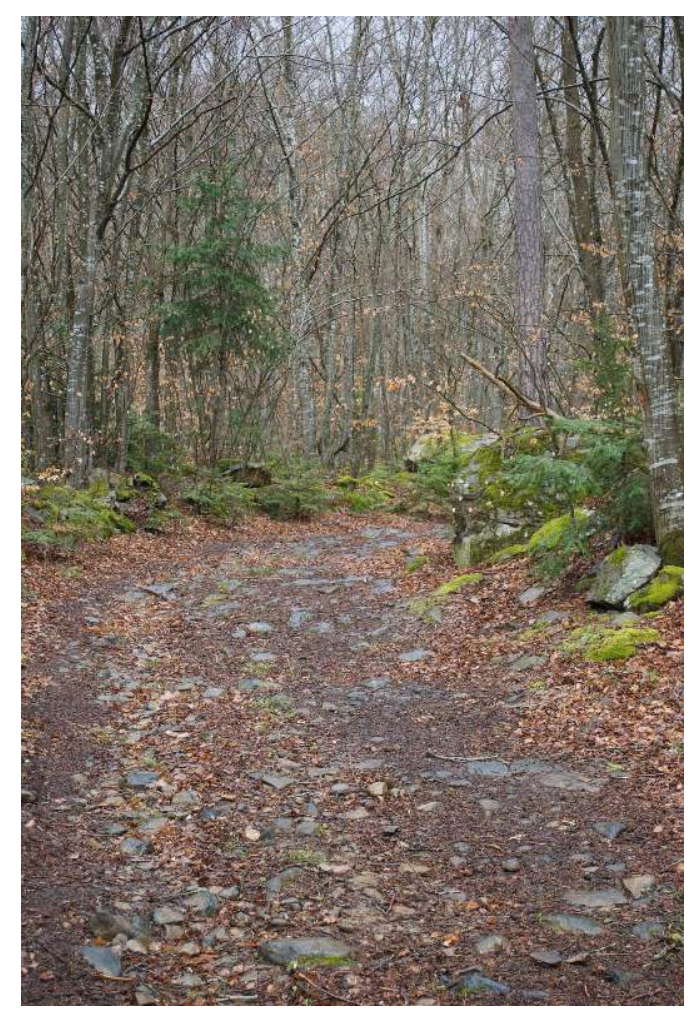

Photo Rym Khene

\section{NOTES}

1. Ces propos sont extraits de l'entretien de Paule Giraud avec Claire Riffard et Rym Khene en avril 2017. Pour "prolonger la promenade », lire aussi l'entretien de Paule Giraud avec Tania Tourjansky, «En approchant Kateb Yacine », paru dans le $n^{\circ} 1$ de la revue Apulée.

2. Amazigh, littéralement « homme libre », fils de Kateb Yacine.

3. Selon la tradition, le prophète Mahomet aurait découpé un pan de son manteau plutôt que de réveiller sa chatte, Muessa, alors qu'il se vêtait au moment d'aller prier.

\section{INDEX}

Mots-clés : Yacine Kateb, Nedjma 